\title{
abTEM: ab Initio Transmission Electron Microscopy Image Simulation
}

Jacob Madsen and Toma Susi

University of Vienna, Vienna, Wien, Austria

Transmission electron microscopy (TEM) is a powerful tool for studying the properties of materials down to the atomic level. In many cases, the quantitative interpretation of images requires simulations based on atomistic structure models. Image simulation for TEM has typically relied on the independent atom model (IAM) that neglects bonding effects [1], which are measurable and important for understanding many systems. The rearrangement of charge due to bonding is directly detectable in HRTEM images and can be crucial for the interpretation of small differences in the atomic contrast [2]. Moreover, the recent rise in popularity of the four-dimensional (4D) scanning TEM has been partially due to promises of unprecedented sensitivity to electromagnetic fields [3]; however, such data call for rigorous theoretical interpretation through simulations.

Since all electrons and the nuclear cores contribute to the scattering potential, simulations that go beyond the IAM have relied on computationally highly demanding all-electron calculations. We have developed a method to generate $a b$ initio electrostatic potentials when describing the core electrons by projector functions. Combined with an interface to quantitative image simulations, this implementation enables an easy and fast means to model electron scattering. By comparing simulated transmission electron microscopy images and diffraction patterns to experimental data, we can show an accuracy equivalent to earlier all-electron calculations at a much lower computational cost [4].

We have now implemented this method in a freely available open-source program based on Python, dubbed "abTEM" for ab initio Transmission Electron Microscopy. abTEM integrates directly with two popular open-source Python codes: the Atomic Simulation Environment (ASE) [5] for setting up atomistic models, and GPAW for calculating electrostatic potentials based on the real-space projector-augmented wave implementation of density functional theory [6]. A highly efficient first-principles description of van der Waals interactions can be included via the libvdwxc library [7].

abTEM is designed for accuracy and includes up to the second-order expansion of the multislice solution of the high-energy Schrödinger equation and uses the correct integration of the sub-slicing of the threedimensional potential [8]. Due to integration with ASE, it is straightforward to model temperature in the frozen phonon approximation using realistic configurations from molecular dynamics simulations. abTEM works as a standard full-featured multislice simulation program, and we have additionally implemented features for speeding up simulations at some cost to accuracy. In particular, abTEM features a GPU-accelerated implementation of the recently developed PRISM algorithm for STEM simulations [9].

Due to its object-oriented modular structure where each part of a simulation is implemented as a separate object, abTEM is highly customizable. It fits nicely into the rest of the Python world by using NumPy arrays for CPU calculations and the NumPy compatible CuPy arrays for fast GPU calculations. This provides seamless access to the full suite of tools in scientific Python, thus providing a single simulation/analysis/visualization framework. While a single graphical user interface (GUI) would be too rigid for our design goals, we include examples of application-specific GUIs to automate common simulation tasks by drawing on projects such as Jupyter. 
We demonstrate the capabilities of our method by modeling twisted bilayer graphene, which has recently received considerable attention due to its extraordinary and highly tunable properties [10]. This system requires on the order of a thousand atoms to represent in simulations, which, to our knowledge, is the largest system ever to be used in a quantitative electron microscopy image simulation that includes an accurate representation of bonding effects. Figs. 1 and 2 show a comparison of the electrostatic potential from the IAM and our method. The differences are subtle due to the large contribution of the cores; hence a great deal of care needs to be taken when extracting charge rearrangements solely based on 4D-STEM measurements.

More information about abTEM is available online [11, 12].
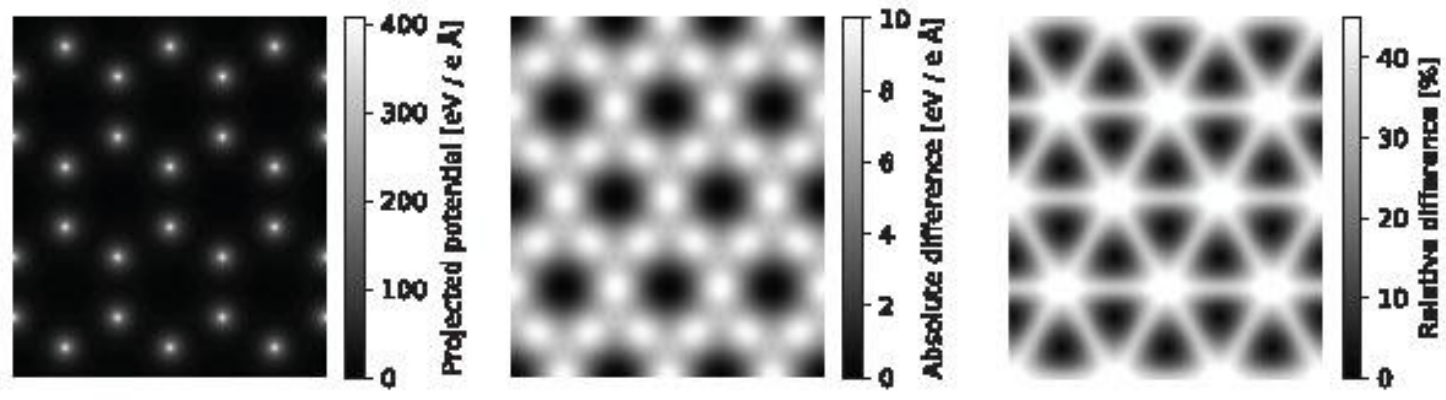

Figure 1. The projected electrostatic potential of a single sheet of graphene calculated with our method, VDFT, and how it differs from the equivalent IAM potential, VIAM. The absolute and relative differences are respectively calculated as VIAM - VDFT, and (VIAM - VDFT) / VIAM. The chemical bonding concentrates additional electron density between the atoms, reducing the potential in these regions due to the resulting additional screening from the core charges.

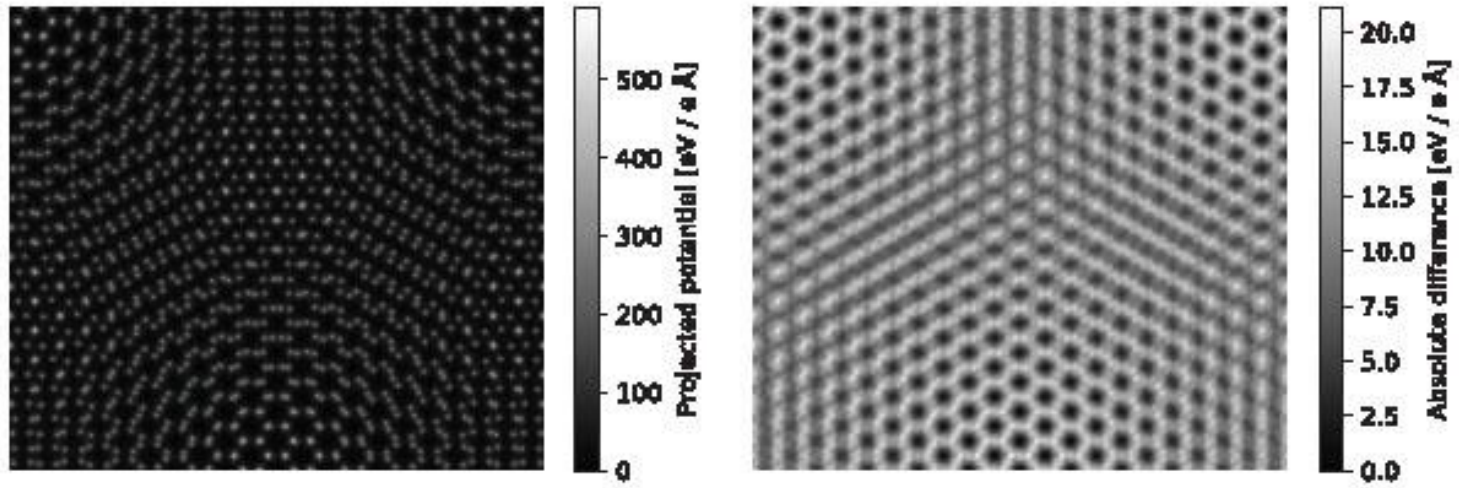

Figure 2. The projected electrostatic potential of twisted bilayer graphene calculated with our method and how this differs from the IAM, shown as described in Fig. 1. The main contribution to the difference is from intralayer bonds; however, further analysis also reveals some effect on the potential due to the interlayer interaction.

\section{References}

[1] E.J. Kirkland, Advanced Computing in Electron Microscopy, $2^{\text {nd }}$ edition, Springer US

[2] S. Kurasch, Beilstein Journal of Nanotechnology 2 (2011) 394

[3] C. Ophus, Microscopy and Microanalysis 25 (2017) 563

[4] T. Susi et al., Ultramicroscopy 197 (2019) 16 
[5] A.H. Larsen et al., Journal of Physics: Condensed Matter 29 (2017) 273002

[6] J.J. Mortensen et al., Physical Review B 71 (2005) 035109

[7] A.H. Larsen et al., Modelling and Simulation in Matererials Science and Engineering 25 (2017) 065004

[8] I. Lobato and D. van Dyck, Ultramicroscopy 156 (2015) 9

[9] C. Ophus, Advanced Structural and Chemical Imaging 3 (2017) 13

[10] M. Yankowitz et al., Science 363 (2018) 1059

[11] https://github.com/jacobjma/abTEM

[12] The work was supported by the European Research Council (ERC) Grant No. 756277-ATMEN. 